\title{
Lecture II: Is regional anaesthesia worthy with hospital business?
}

Patrick Narchi ${ }^{1, *}$

${ }^{1}$ Anesthesia Department, Centre Clinical, 16800 Soyaux, France.* patrick.narchi@yahoo.fr

The direct costs of anesthesia and analgesia (including anesthesia fee) represents only $2-4 \%$ of the total cost of a surgical procedure. the type of surgical procedure or its duration can make regional anesthesia less expensive or more expensive than general anesthesia. However, optimization of the total time spent inside the OR represents the main factor of the total bill (50-60\%). Such a reduction in the total OR time leads to a significant decrease in personnel costs which represents $70 \%$ of the cost. Thus, scheduling, organizing flow of patients through the OR is the first improving factor to control. In this field, regional anesthesia can significantly reduce this major cost factor through multiple mechanisms: (1) setting a "block room" to perform blocks in a dedicated space outside the OR for high output short surgical procedures (hand surgery cases) improves the efficiency of the OR by $56 \%$. (2) Regional anesthesia techniques reduce the duration of stay in PACU compared to general anesthesia and even allows more easily to encourage PACU bypass (10-82\% of patients). (3) Regional anesthesia and analgesia significantly increase the percentage of surgical procedures which are performed on an ambulatory basis, compared to general anesthesia. Such a shift towards ambulatory surgery leads to a major decrease in nursing budgets for the hospital. (4) Finally, all ERAS surgical procedures require somehow the administration of local anesthetics which can go from intravenous lidocaine (associated to general anesthesia), to infiltration techniques, to nerve blocks. In conclusion, Regional anesthesia techniques, when combined to a strict optimisation of the management of patients flow throughout the whole process, are 2 major factors that significantly improve hospital business. 